\title{
Health Care Workers' Expectations and Empathy toward Patients in Abusive Relationships
}

\author{
Christina Nicolaidis, MD, MPH, MaryAnn Curry, RN, DNSc, and \\ Martha Gerrity, MD, MPH, PhD
}

Purpose: To understand attitudes that may affect health care workers' ability to provide appropriate long-term care for patients who stay with abusive partners.

Methods: We surveyed 278 health care workers in 31 primary care practices before their participation in an educational intervention.

Results: More than half of participants (51\% to 60\%) found it easy to empathize with a patient who decided to remain in an abusive relationship if the patient was described as poor or disabled, but few (25\% to 39\%) could empathize if the patient was described as educated or financially secure. A majority (57\% to 59\%) agreed with a statement meant to assess unrealistic expectations. ("A provider's responsibility includes making sure a patient gets to a shelter right away if he or she discloses abuse.") Participants who agreed with this statement had more difficulty empathizing with patients who decided to stay with an abusive partner $(P=.0045)$.

Conclusions: Training efforts must focus on screening and on helping providers develop more realistic expectations about the complex nature of leaving an abusive relationship. Health care workers need a better understanding of the barriers patients face and why patients may choose to remain in abusive relationships, even in the absence of economic or health limitations. (J Am Board Fam Pract 2005; 18:159-65.)

Most studies of health care workers' attitudes and behaviors regarding intimate partner violence (IPV) have focused on screening practices and on barriers to screening. ${ }^{1-13}$ However, the typical pattern of IPV is that patients stay in abusive relationships long after the initial screening or disclosure takes place. Primary care providers and their staff must be able to provide long-term continuity care to patients who remain in abusive relationships.

Qualitative data suggest that women in abusive relationships often believe health care workers have unrealistic expectations about their ability or desire to leave an abusive relationship and that they have

Submitted, revised, 15 February 2005.

From the Division of General Internal Medicine (CN, MG) and School of Nursing (MAC), Oregon Health and Science University, and Portland Veterans Affairs Medical Center (MG), Portland. Address correspondence to Christina Nicolaidis, MD, MPH, L475, Division of General Internal Medicine, Oregon Health and Science University, Portland, OR 97215 (e-mail: nicolaid@ohsu.edu).

This work was funded by grant 2001-331 from the Northwest Health Foundation (to CN).

Presented at the Annual Meeting of the Society of General Internal Medicine; 2004 May 12-15; Chicago, Illinois; and at the National Conference on Domestic Violence and Healthcare; 2004 Oct 20-22; Boston, Massachusetts. negative attitudes toward patients who stay with abusive partners or who do not leave the relationship on the provider's timeline. ${ }^{14}$ Additional qualitative studies with IPV survivors have noted important themes about the negative or judgmental attitudes of health care providers. ${ }^{15,16}$ Women do not disclose abuse because they fear that a provider will look down on them or be judgmental. ${ }^{17}$ Likewise, they indicate that not being able to follow a providers' advice to leave an abusive relationship is a reason for not returning for further care. ${ }^{18}$

Despite the wealth of information on health care workers' barriers to assessing and discussing IPV, little is known about attitudes that may affect a provider's ability to provide appropriate long-term care or to empathize with patients who remain in abusive relationships. Our objective was to identify attitudes and characteristics associated with health care workers' ability to empathize with patients.

\section{Methods}

\section{Setting, Participants, and Recruitment}

We conducted a cross-sectional survey at $31 \mathrm{com}-$ munity-based primary care practices in Washing- 
ton County, Oregon. These practices agreed to participate in an educational intervention about IPV. We used responses from the preintervention survey to test whether specific attitudes or characteristics were associated with health care workers' ability to empathize with patients. The development of the survey and changes in survey responses after the intervention are reported elsewhere. ${ }^{19} \mathrm{We}$ offered the training program to the 90 family medicine, general internal medicine, and obstetrics/gynecology practices located in Washington county via letters, phone calls, and in-person visits. In most cases, the office manager or medical director of the practice decided whether or not the clinic would participate in the training. Because of the heterogeneous nature of these practices, we allowed the management of each practice to decide which employees would participate in the training. We were unable to collect data on how many employees were asked by management to participate but declined.

\section{Data Collection}

As reported elsewhere, we developed the Attitudes Toward Survivors of IPV survey (ATSI) ${ }^{19}$ because were unable to find previously validated instruments that adequately assessed health care workers' attitudes or empathy. Participants completed the ATSI before their scheduled training session. The ATSI addresses 8 domains of IPV-related attitudes and practices: responsibility regarding assessment for IPV, responsibility regarding counseling and management of IPV, respect for patient autonomy, empathy toward patients who choose to remain in abusive relationships, barriers to screening, confidence, self-reported behaviors, and knowledge. Part 1 of the survey consisted of statements beginning with "A primary care provider's responsibility includes. . " Participants responded to each item using a 5-point Likert scale ranging from 1 ("strongly disagree") to 5 ("strongly agree"). Included were 3 questions regarding a primary care providers' responsibility to assess for IPV in patients presenting for 1) routine health maintenance, 2) injury, and 3) chronic pain and 3 questions about appropriate counseling practices. To assess for potential social desirability bias, we included a statement about ". . . asking about domestic violence at every visit," which we would not consider to be a primary care provider's responsibility. To assess for unrealistic expectations regarding the natural his- tory of IPV, we also included the statement: "A primary care provider's responsibility includes making sure a patient gets to a shelter right away if he or she discloses abuse." An additional item about "telling a patient he or she needs to leave an abusive relationship" was included to further measure respect for patient autonomy.

Part 2 of the survey asked participants to "mark how easy or difficult it would be to empathize with each of the following patients' decision to remain in an abusive relationship." Participants were given 8 scenarios with varying sex, sexual preference, marital status, income, education, and disability. Participants responded using a 5-point Likert scale, ranging from 1 ("very difficult") to 5 ("very easy").

A section on self-reported behavior listed different presenting complaints and asked participants how often they had assessed for IPV when seeing patients in the past month with each condition. Again, to assess for potential social desirability bias, we purposely included one scenario (coronary artery disease) in which we did not feel it was reasonable to always ask about IPV. Responses were rated on a 5 -point Likert scale ranging from 1 ("never") to 5 ("always"). Participants were instructed to mark "not applicable" if they do not interview patients or if they have not seen a patient with this condition in the past month. Additional survey sections addressed barriers to assessing and managing IPV, confidence, experience, availability of resources, knowledge, prior IPV training, and demographic characteristics.

\section{Analysis}

We dichotomized responses regarding sense of responsibility, unrealistic expectations, empathy, and self-reported behaviors. We created scale scores for each theoretical domain by summing the scores for items in the domain and dividing by the number of items answered. Scores for the responsibility to assess, responsibility to counsel, empathy, barriers, and self-reported behavior scales could range from 1 to 5 . Scores for the confidence scale could range from 1 to 3 and scores for the knowledge scale could range from 0 to 4 . Questions meant to assess social desirability bias were not included in the summary scores. We assessed internal reliability of each scale using Cronbach $\alpha$. We used $t$ tests, $\chi^{2}$ tests, and Pearson correlations to assess bivariate associations. We used a 2-way analysis of variance to test the association between unrealistic expecta- 
tions and empathy while adjusting for professional role (provider versus support staff).

We performed statistical analyses using STATA software (ver 6.0; Stata Corporation, College Station, TX). The study was approved by Oregon Health \& Science University's human subjects committee. A grant from the Northwest Health Foundation funded the study.

\section{Results}

We collected surveys from 278 participants. Demographic data are shown in Table 1 . Seventy responses $(25 \%)$ were from primary care providers (physicians, nurse practitioners, or physician assistants); 122 (44\%) from medical support staff (nurses, medical assistants, or social workers); 56 (20\%) from administrative staff (reception or billing); and 21 (8\%) from "other" employees (eg, community outreach workers). Sixty-two percent had received at least some form of IPV training in the past. Fifty-nine percent stated that they, a family member, or close friend had experienced IPV.

Table 1. Demographic Characteristics for the 278 Participants

\begin{tabular}{lc}
\hline Characteristics & $\mathrm{N}(\%)$ \\
\hline Professional role* & \\
Primary care providers & $70(25)$ \\
Medical support staff & $122(44)$ \\
Administrative staff & $56(20)$ \\
Other employees & $30(8)$ \\
Unknown & $9(3)$ \\
Medical specialty (providers only) & \\
Family medicine & $32(46)$ \\
Internal medicine & $19(27)$ \\
Obstetrics/gynecology & $12(17)$ \\
Other & $7(10)$ \\
Male sex $\dagger$ & $26(9)$ \\
Prior domestic violence training & \\
Self, family member, or close friend with & $123(62)$ \\
history of domestic violence & $134(59)$ \\
\hline
\end{tabular}

* Primary care providers include 48 physicians, 17 nurse practitioners, and 5 physician assistants; medical support staff include nurses, medical assistants, and technicians; administrative staff include clinic managers, receptionists, records clerks, and billing clerks; and other employees include social workers and community outreach workers. Nine nonphysician employees did not specify a clinical role.

† Twenty of the 48 (42\%) physicians were male.

₹ Only 199 and 226 participants responded to questions about prior domestic violence training or personal experience with domestic violence, respectively.

\section{Health Care Workers' Attitudes and Self-Reported Behaviors}

A majority of participants agreed that it is a primary care provider's responsibility to ask about IPV when seeing patients for health maintenance visits (66\%), chronic pain (56\%), or injuries (62\%). Summary scores for responsibility to assess for IPV had a mean of 3.6 (SD 0.8) on a scale of 1 to 5. More than $75 \%$ of participants ( $78 \%$ to $92 \%$ ) agreed with the 3 items about appropriate counseling practices. Only $15 \%$ agreed with the statement about assessing for IPV at every visit. However, a majority $(58 \%)$ also agreed with the statement meant to assess unrealistic expectations (Table 2). Primary care providers (physicians and midlevel practitioners) had lower rates of agreement with the statement about unrealistic expectations than did other staff ( $40 \%$ vs. $64 \% ; P=.001)$. There was no significant difference between providers and other staff in responses to items about responsibility to assess or manage IPV.

Despite the high level of agreement with statements about responsibility to assess for IPV, few participants $(12 \%$ to $35 \%)$ stated that they had asked about IPV "nearly always" or "always" in the past month when seeing patients with injuries, chronic pelvic pain, irritable bowel syndrome, headaches, depression or anxiety, routine health maintenance, or prenatal care (Table 3). Only one person stated that she routinely assessed for IPV when seeing patients for coronary artery disease. The self-reported behavior scale had a mean of 2.4 (S.D. 1.0). Participants' sense of responsibility to assess for IPV was associated with their self-reported behaviors $(\mathrm{r}=0.40 ; P<.0001)$.

A majority of participants (51\% to $60 \%$ ) stated that it was "easy" or "very easy" to empathize with patients who chose to remain in abusive relationship when the patients were described as poor, uneducated, depressed, or physically disabled. However, few (25\% to $39 \%$ ) could empathize when the patient was described as educated, middle-class, professional, or having a steady income, regardless of patient sex, marital status, or sexual orientation. Providers were more likely than other staff to empathize with patients who were described as poor or ill. There was no significant difference by professional role in responses to items describing patients of higher socioeconomic status (Figure 1). The empathy scale had a mean of 3.0 (SD 0.9). Primary care providers had higher overall empathy scores 
Table 2. Percentage of Healthcare Workers Agreeing with Items about a Primary Care Provider's Responsibility to Assess for or Counsel about Intimate Partner Violence

This section was preceded by the statement: "Primary care providers are asked to do increasingly more for patients in increasingly less time. For each of the statements below please mark your level of agreement regarding what should be expected of a primary care provider. The provider's responsibility includes:"

Screening female patients for domestic violence at every routine health maintenance visit.

Asking all patients with chronic pain about the possibility of domestic violence. (Responsibility to assess for IPV)

Asking about domestic violence any time an injury is noticed, regardless of the stated cause.

(Responsibility to assess for IPV)

Asking about domestic violence at every visit. (Social desirability)

Making sure a patient gets to a shelter right away if he or she discloses abuse. (Unrealistic expectations/ lack of respect for autonomy)

Telling a patient that an abusive partner's behavior is not acceptable. (Responsibility to manage IPV)

Telling a patient that a particular relationship is harmful to his or her health. (Responsibility to manage IPV)

Following-up with a patient after making a referral to a domestic violence agency. (Responsibility to manage IPV)

Telling a patient he or she needs to leave an abusive relationship. (Lack of respect for autonomy)

than other employees (3.2 vs 3.0, respectively; $P=$ $.04)$.

\section{Relationship between Expectations and Empathy}

Participants who agreed with the statement meant to assess unrealistic expectations ("A primary care provider's responsibility includes making sure a patient gets to a shelter right away if he or she discloses abuse") had lower total empathy scores than those who were neutral or did not agree with that statement (mean 2.9 vs $3.2 ; P=.0045$ ). The ability to empathize with patients' choices was not related to sex, prior IPV training, personal experience with IPV, responsibility regarding IPV assessments and management, self-reported behaviors, confidence, knowledge, or barriers. In a 2-way analysis of variance, the association between unrealistic expectations and empathy score remained significant $(P=$ .02) after adjustment for professional role. Results did not significantly change when participants who agreed with statements meant to indicate social desirability bias were excluded.

\section{Table 3. Responses to Items Regarding Self-Reported Behavior}

\begin{tabular}{|c|c|c|}
\hline $\begin{array}{l}\text { Type of visit } \\
\text { Participants were instructed: "Please indicate how often you have } \\
\text { asked a patient about the possibility of Domestic Violence when you } \\
\text { saw any of the following conditions in the last month. If you have } \\
\text { not seen this condition in the past month, mark N/A. Please skip to } \\
\text { the next section if you do not interview patients." }\end{array}$ & $\begin{array}{l}\text { Number of respondents } \\
\text { answering item* }\end{array}$ & $\begin{array}{c}\text { "Nearly always" or "always" } \\
\text { assess for IPV } \\
{[\%(\mathrm{~N})]}\end{array}$ \\
\hline Injuries (bruises, lacerations, etc.) & 114 & $35(40)$ \\
\hline Chronic pelvic pain & 116 & $18(22)$ \\
\hline Irritable bowel syndrome & 112 & $13(14)$ \\
\hline Headaches & 129 & $12(15)$ \\
\hline Depression/anxiety & 134 & $24(32)$ \\
\hline Coronary artery disease $^{\dagger}$ & 104 & $1(1)$ \\
\hline Routine health maintenance exam & 121 & $26(32)$ \\
\hline Pre-natal care & 89 & $31(28)$ \\
\hline
\end{tabular}

* The number of respondents varies by item because not all participants interviewed patients or saw these conditions in the past month.

${ }^{\dagger}$ The item regarding coronary artery disease was intended to measure social desirability bias and was not included in the summary score for the self-reported behaviors scale. 
Table 4. Bivariate Analyses Assessing the Association between Empathy and Healthcare Workers' Characteristics

\begin{tabular}{|c|c|c|}
\hline Characteristics & $\begin{array}{l}\text { Mean } \\
\text { Empathy } \\
\text { Score }\end{array}$ & $P$ value \\
\hline \multicolumn{3}{|l|}{ Professional role } \\
\hline Providers & 3.2 & \multirow[t]{2}{*}{.04} \\
\hline Other staff & 3.0 & \\
\hline \multicolumn{3}{|l|}{ Unrealistic Expectations } \\
\hline Agree or strongly agree & 2.9 & \multirow[t]{2}{*}{.0045} \\
\hline Strongly disagree to neutral & 3.2 & \\
\hline \multicolumn{3}{|l|}{ Other Characteristics } \\
\hline \multicolumn{3}{|c|}{$\begin{array}{l}\text { The following variables did not correlate with empathy: sex, } \\
\text { personal experience with IPV, prior IPV training, sense of } \\
\text { responsibility to assess for IPV, sense of responsibility to } \\
\text { manage IPV, confidence, barriers, knowledge, and self- } \\
\text { reported behavior. }\end{array}$} \\
\hline
\end{tabular}

\section{Discussion}

A majority of health care workers endorsed a statement that indicated an unrealistic expectation of getting a patient to a shelter right away after the patient discloses a history of abuse. Agreement with this statement was associated with greater difficulty empathizing with patients who remain with abusive partners. Beliefs or practices about screening did not correlate with empathy.

Most health care workers, regardless of professional role, found it difficult to empathize with patients remaining in abusive relationships when such patients were described as educated, healthy, or financially secure. When patients were described as poor or disabled, primary care providers found it easier to empathize with patients staying in abusive relationships than did other employees, including nurses, medical assistants, administrative staff, and outreach workers. Primary care providers were also less likely to agree with the statement about the unrealistic expectation of getting a patient to a shelter right away after a disclosure of abuse. Providers may have a more comprehensive knowledge of patients over time than the other personnel and are perhaps more "seasoned" to understand the complexities of IPV, at least for patients of lower socioeconomic status. Providers' ability to understand these complexities, however, seems to diminish for patients of higher socioeconomic status. Furthermore, the association between unrealistic expectations and empathy persisted, even after adjustment for professional role.
This study has several important limitations. First, we used a convenience sample of practices that agreed to participate in a domestic violence educational intervention. Although most employees did not participate in the clinic's decision regarding the training, it is possible that the employees of these clinics are different from those of clinics who were not interested in gaining further IPV training. We do not know how many health care workers could have been eligible to participate, so we cannot determine response rate. Furthermore, it is possible that employees who participated in the IPV trainings were likely to be different from other employees at the same clinics.

We were only able to measure self-reported behaviors of health care workers, not actual practices or patient perceptions. Other surveys of health care workers' attitudes about IPV have not assessed sense of responsibility, unrealistic expectations or empathy with patients choices, so we developed items to assess these constructs. As presented elsewhere, ${ }^{19}$ each scale had fair to excellent internal reliability and was responsive to change. However, further research is needed to establish the validity of these measures.

We only used one question to assess for unrealistic expectations, "A primary care provider's responsibility includes making sure a patient gets to a shelter right away if he or she discloses abuse." That item is part of the ATSI scale measuring lack of respect for patient autonomy. The other item meant to assess lack of respect for patient autonomy ("A primary care provider's responsibility includes telling a patient s/he should leave an abusive relationship") did not correlate with empathy. Further research is needed to understand the potential influence of controlling attitudes or behaviors on health care workers' ability to counsel or empathize with patients in abusive relationships.

Despite these limitations, our study has important implications. Domestic violence training efforts must focus not only on increasing screening rates but also on developing more realistic expectations about the typical course of abusive relationships. Health care workers need to better understand why patients may stay with abusive partners, especially in the absence of economic or health limitations. Such reasons may include lack of recognition of the abuse, a commitment to the relationship, the belief that the partner will change, erosion of self-esteem, lack of options, and the 
Paticnt Characteristics*:

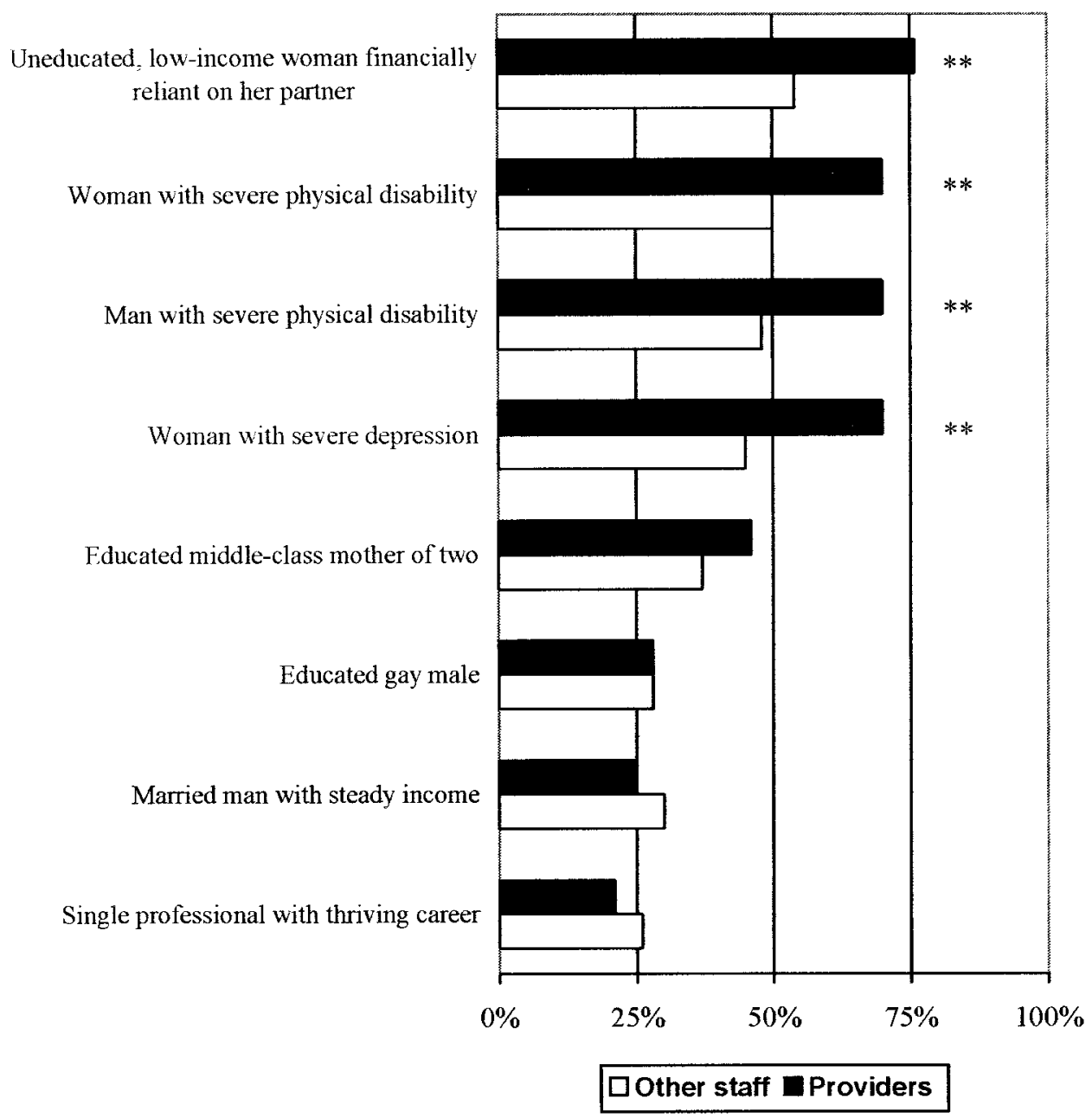

Figure 1. Participants stating it is "easy" or "very easy" to empathize with a patient who remained in an abusive relationship.

*The section about empathy was preceded by the following statement: "Health care providers generally find it easier to empathize with some people's choices than others. Imagine a patient is choosing to remain in an abusive relationship. For each of the patient types listed below, please mark how easy or difficult it is for you to empathize with their decision to remain in the abusive relationship."

$* * P<.001$ for difference between providers and other staff.

danger associated with leaving an abusive relationship. ${ }^{14}$ Particular attention is needed to dispel providers' stereotypes that may affect their inability to empathize with IPV victims of higher socioeconomic status. Nurses and medical assistants may play important roles in screening and counseling patients about IPV. ${ }^{20,21}$ However, our study indicates that support staff may have even greater training needs than primary care providers. As reported elsewhere, we have found that the Voices of Survivors program is effective in changing unrealistic expectations, the ability to empathize with patients' choices, and self-reported behaviors. ${ }^{19}$ However, further research is needed to determine the effect of changing health care workers' attitudes and empathy on actual behavior, patient satisfaction, or patient outcomes.

We thank Jim Winkle for excellent work in recruitment, training, and data collection and the Domestic Violence Resource Center of Washington County for collaboration with this project. We also thank Sarah Zwelle-Burke for assistance with grant-writing, Don Austin for assistance with the project design, Chiquita Rollins for manuscript suggestions, and Cari Gandrud 
for administrative assistance. None of the authors has any conflicts of interest to disclose.

\section{References}

1. Jonassen JA, Mazor KM. Identification of physician and patient attributes that influence the likelihood of screening for intimate partner violence. Acad Med 2003;78(10 Suppl):S20-3.

2. Sitterding HA, Adera T, Shields-Fobbs E. Spouse/ partner violence education as a predictor of screening practices among physicians. J Contin Educ Health Prof 2003;23:54-63.

3. Guedes A, Bott S, Cuca Y. Integrating systematic screening for gender-based violence into sexual and reproductive health services: results of a baseline study by the International Planned Parenthood Federation, Western Hemisphere Region. Int J Gynaecol Obstet 2002;78(Suppl 1):S57-63.

4. Davis RE, Harsh KE. Confronting barriers to universal screening for domestic violence. J Prof Nurs 2001;17:313-20.

5. Glass N, Dearwater S, Campbell J. Intimate partner violence screening and intervention: data from eleven Pennsylvania and California community hospital emergency departments. J Emerg Nurs 2001; 27:141-9.

6. Clark KA, Martin SL, Petersen R, et al. Who gets screened during pregnancy for partner violence?. Arch Fam Med 2000;9:1093-9.

7. Waalen J, Goodwin MM, Spitz AM, Petersen R, Saltzman LE. Screening for intimate partner violence by health care providers: barriers and interventions. Am J Prev Med 2000;19:230-7.

8. Rodriguez MA, Bauer HM, McLoughlin E, Grumbach K. Screening and intervention for intimate partner abuse: practices and attitudes of primary care physicians. JAMA 1999;282:468-74.

9. Larkin GL, Hyman KB, Mathias SR, D'Amico F, MacLeod BA. Universal screening for intimate partner violence in the emergency department: importance of patient and provider factors. Ann Emerg Med 1999;33:669-75.

10. Rural health-care providers' attitudes, practices, and training experience regarding intimate partner vio-
lence-West Virginia, March 1997. MMWR Morb Mortal Wkly Rep 1998;47:670-3.

11. McGrath ME, Hogan JW, Peipert JF. A prevalence survey of abuse and screening for abuse in urgent care patients. Obstet Gynecol 1998;91:511-4.

12. McGrath ME, Bettacchi A, Duffy SJ, Peipert JF, Becker BM, St Angelo L. Violence against women: provider barriers to intervention in emergency departments. Acad Emerg Med 1997;4:297-300.

13. Currier GW, Barthauer LM, Begier E, Bruce ML. Training and experience of psychiatric residents in identifying domestic violence. Psychiatr Serv 1996; 47:529-30.

14. Nicolaidis C. The Voices of Survivors documentary: using patient narrative to educate physicians about domestic violence. J Gen Intern Med 2002;17:11724.

15. Chang JC, Decker M, Moracco KE, Martin SL, Petersen R, Frasier PY. What happens when health care providers ask about intimate partner violence? A description of consequences from the perspectives of female survivors. J Am Med Wom Assoc 2003;58: $76-81$.

16. Gerbert B, Johnston K, Caspers N, Bleecker T, Woods A, Rosenbaum A. Experiences of battered women in health care settings: a qualitative study. Women Health. 1996;24:1-17.

17. McCauley J, Yurk R, Jenkins M, Ford D. Inside "Pandora's box": abused women's experiences with clinicians and health services. J Gen Intern Med 1998;13:549-55.

18. Nicolaidis C. The voices of survivors: domestic violence survivors educate physicians [videocassette]. Philadelphia: American College of Physician; 2000.

19. Nicolaidis C, Curry MA, Gerrity M. Measuring the impact of the Voices of Survivors program on healthcare workers' attitudes toward survivors of intimate partner violence. J Gen Intern Med. In press 2005.

20. Campbell J. Family violence and nursing practice. Philadelphia: Lippincott Williams \& Wilkins; 2004.

21. Wiist WH, McFarlane J. The effectiveness of an abuse assessment protocol in public health prenatal clinics. Am J Public Health 1999;89:1217-21. 